\title{
Cost of illness of RSV infection in a middle-income tropical country
}

\author{
Jefferson Antonio Buendía ${ }^{\oplus}$, Diana Guerrero Patino ${ }^{\oplus}$, Denis Sinisterra ${ }^{\oplus}$ \\ Grupo de Investigación en Farmacología y Toxicología (INFARTO). Departamento de Farmacología y Toxicología, Facultad de \\ Medicina, Universidad de Antioquia, Carrera, Medellín, Colombia.
}

\begin{abstract}
Background. Despite the burden of disease of Respiratory syncytial virus (RSV) infection in children, there are important gaps in knowledge about the potential impact in terms of health as well as social and healthcare resources. The aim of this study was to describe the economic burden of RSV in the first two years of life in Colombia.

Methods. We conducted a cost-of-illness study, taking a population prevalence-based approach. A decision tree model was constructed with a time horizon of two years. We defined the following outcomes: death, RSV infection with long term complications, RSV with acute complications, RSV without complications. Inpatient and outpatient costs were collected directly from medical invoices of patients who attended a tertiary referral hospital. Results. The mean cost per patient with an RSV infection was US\$ 178.35 CI 95\% (30.7-541.67 US\$). The total cost of RSV infection in children less than 2 years in Colombia was US \$ 64443616 per year (CI 95\% US\$11 092902 - US\$195 722 867). In the probabilistic sensitivity analysis, the mean cost per patient with RSV infection was only sensitive to changes in the cost of recurrent wheezing, cost of outpatient visits and cost of hospitalizations.

Conclusion. The infection by RSV in Colombia generates a high economic burden on the health system. Generating comprehensive data on healthcare resource use and costs associated with RSV will help to provide valuable information for the development of cost-effectiveness models, and help guide prevention strategies against RSV.
\end{abstract}

Key words: cost, respiratory syncytial virus, Colombia.

Respiratory syncytial virus (RSV) is the most frequent cause of bronchiolitis worldwide.1 In 2005, 33. ${ }^{1}$ million episodes of RSV infections worldwide resulted in about 3.2 million hospital admissions, and 59,600 in-hospital deaths in children younger than five years. ${ }^{2}$ Worldwide, RSV infection is the second cause of postnatal infant death after malaria, causing 137,000 deaths each year (equal to $6.7 \%$ of all newborn deaths). ${ }^{3}$

Despite the increased risk of RSV in children and the frequency of their complications, there is an

Jefferson Antonio Buendía jefferson.buendia@gmail.com

Received 2nd March 2020, revised 17th August 2020, 10th November 2020, 8th January 2021, accepted 8th February 2021. important gap in the knowledge concerning the potential impact in terms of health as well as social and healthcare resources. Most of the economic studies focused on assessing the efficiency of palivizumab for the prophylaxis of RSV infection and have not properly evaluated the impact of this infection beyond the acute episode phase..$^{4-7}$ Many of these studies have been designed from the perspective of the payer and not from a social perspective, without including indirect costs such as those associated with job loss and family expenses. Likewise, no studies have evaluated the economic impact of such an infection in developing countries; in which the problem in terms of morbidity and mortality is growing. ${ }^{8}$ The aim of this study was to describe the economic burden of RSV infection in the first two years of life in Colombia. 


\section{Material and Methods}

\section{Choice of model}

We conducted a cost-of-illness study, taking a population prevalence-based approach. A decision tree model was constructed to estimate the cost of each episode of RSV infection (Fig. 1). The reason for using a decision tree to estimate the expected cost per patient is that this analytical approach reduces the possible selection bias in the population studied that over represents or underestimates both the frequency of the outcome and the derived costs. With this structure, the only factor that weighs the cost are the probabilities of the analytical model and not the chance that such events may or may not occur in the population secondary to selection bias. ${ }^{9-12}$ The study protocol and their informed consent was reviewed and approved by the Institutional Review Board of Clinica Somer (No 281015) and the University of Antioquia (No 18/2015).

\section{Target population}

Children younger than two years of age admitted to the pediatric ward with a diagnosis of RSV infection were included in the study. Patients older than two years, without lower respiratory compromise, with positive bacterial cultures on admission, confirmed whooping cough or those referred from another hospital center were excluded.

\section{Setting and location}

Rionegro is a city and municipality in Antioquia Department, Colombia, located in the subregion of Eastern Antioquia, at an average elevation of 2,125 meters above sea level. The average annual precipitation varies between 1,800 and 2,500 millimeters with an average temperature of $17^{\circ} \mathrm{C}$, with a peak in the presentation of RSV between March and April. ${ }^{13}$ The municipality of Rionegro has a total population of 101,046 inhabitants, the sixth largest populated area in Antioquia, with two tertiary referral hospital. ${ }^{14}$ Colombia's health system is composed by a social security sector and a private sector. The basis of the system is the General Social Security Health System, which has two plans, contributory and subsidized; all with a decentralized referral mechanism. The contributory regimen covers salaried workers, pensioners, and independent workers, with the subsidized plan covering anyone who cannot pay. The National Health Authority's functions under the system include an increase in the quality of health care and supervision, surveillance, and control of health insurance. Enrollment in the General Social

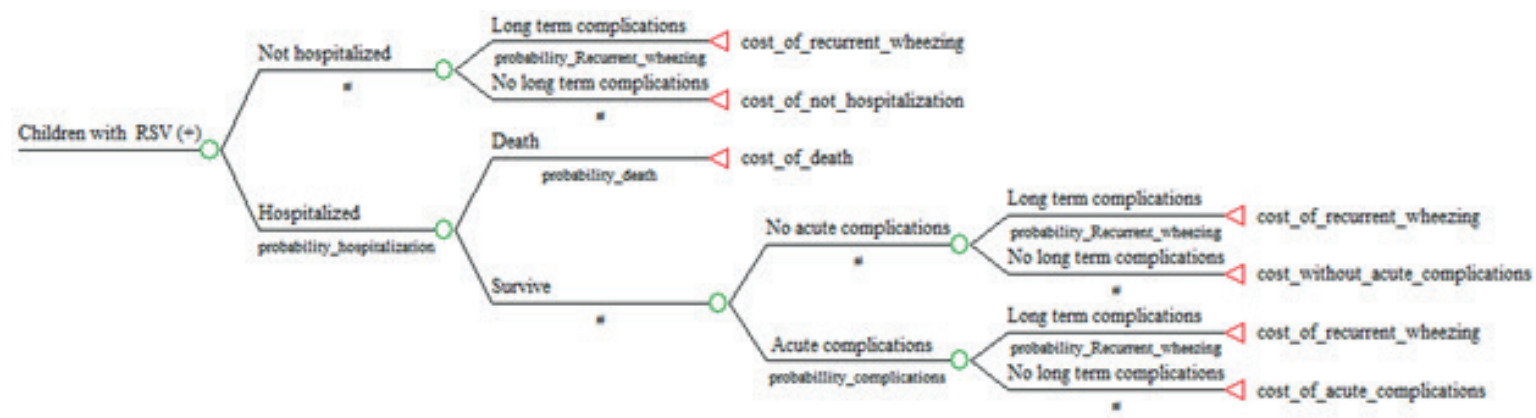

Fig. 1. Decision tree model.

cRecurrent_Wheezing: cost of RSV patient with recurrent wheezing

cNoHosp: cost of RSV patient Not hospitalized

cWithoutComp: cost of RSV patient hospitalized without complications

cComplications: cost of RSV patient hospitalized with complications

cdeath: cost of RSV patient death

pHosp: probability of hospitalization

pCompl: probability of complications

pdeath: probability of death

pRecurrent Wheezing: probability of recurrent wheezing 
Security Health System is obligatory and is handled through public or private health promotion agencies. Health care is provided by institutional health service providers. Those who can afford to purchase health insurance coverage on their own and who can pay for any uncovered fees out-of-pocket use the private sector.

\section{Study perspective and Time horizon}

We conducted a cost-of-illness study, taking a population prevalence-based approach. A decision tree model was constructed with a time horizon of two years. This analysis was performed from a societal perspective including direct and indirect costs. No discount rate was applied to the cost due to the short time horizon evaluated.

\section{Choice of health outcomes}

We defined the following outcomes according to the natural history of RSV infection: death, RSV infection with long term complications (recurrent wheezing), RSV infection only with acute complications, RSV infection without complications. In this model, the development of recurrent wheezing was assumed as the only long-term complication; since it is the most frequent respiratory complication in the first two years after the first infection. ${ }^{15}$ Among the acute complications included: pneumonia, atelectasis, sepsis, pleural effusions, and pneumothoraxes. ${ }^{16}$

\section{Measurement and evaluation of probabilities of model.}

The probabilities of the model were obtained from literature and are listed in Table I. The search was performed in February 2019 and was limited to primary literature published in the English or Spanish language, human subjects, and children (birth to 5 years). The following engines were searched for the periods specified: MEDLINE from 1950 on, EMBASE from 1974 on, BIREME from 1980 on. To avoid missing any articles published we performed a search using Google search engine and we reviewed the first 100 results returned. Terms for these database searches included keywords closely matching the relevant medical field headings: respiratory syncytial virus, and respiratory syncytial pneumovirus. The authors (JAB, DG) reviewed all potentially relevant references independently and selected relevant publications. The study inclusion criteria were studies: (1) reporting the frequency or incidence of outcomes during the episode of community acquired, medically attended, severe RSV infection in children < 2 years for data analysis. (2) reporting data on laboratory confirmed diagnosis of RSV through enzyme-linked immunosorbent assay, polymerase chain reaction (PCR; Multiplex), immunofluorescence (IF), culture, direct fluorescent antibody test (DFA), or by relevant International Classification of diseases-9 (ICD9) diagnosis codes. Population estimates of cases with RSV infection aged $<2$ years, was obtained from the National Institute of Health of Colombia (1).

\section{Estimating resources and costs}

To estimate resources and cost we conducted a prospective study in infants under two years of age admitted to the tertiary centers, in Rionegro due to an RSV infection (ICD-10 code: J21.0, according to the national clinical guideline of bronchiolitis ${ }^{17}$ ) from January 2015 to December 2016. Although the costs were derived from tertiary centers of Rionegro, all values as

Table I. Probabilities used in the model.

\begin{tabular}{lccc}
\hline & Probability & CI 95\% & Distribution \\
\hline Hospitalization & $0.014^{15}$ & $0.008-0.021$ & Beta \\
Death & $0.009^{15}$ & $0.003-0.014$ & \\
Acute complications & $0.144^{15}$ & $0.075-0.195$ & $0.206-0.357$ \\
Recurrent wheezing & $0.281^{13}$ & & \\
\hline
\end{tabular}


detailed below were subjected to sensitivity analysis with a certain range and distribution in order to increase the external validity of our results.

Inclusion criteria were defined as children younger than two years of age admitted to the pediatric ward with a diagnosis of RSV confirmed using direct immunofluorescence (Light Diagnostics TM Respiratory Panel 1 DFA, Merck-Millipore Laboratory). Patients older than two years, without lower respiratory compromise, with positive bacterial cultures on admission, confirmed whooping cough, and those referred from another hospital center were excluded. After receiving informed consent from the parent or caregiver, the patient was interviewed and their electronic medical records were reviewed. We collected the following variables: age, sex, weight, height, signs, and symptoms at admission (e.g. fever, chest indrawing, chest auscultation abnormalities like rhonchi or crepitation), history of prematurity, bronchopulmonary dysplasia confirmed by a specialist physician on discharge from the neonatology unit, comorbidities (congenital heart disease, neurological disease), results of chest X-rays or other medical test, drugs and other treatments, and complications (pneumonia, atelectasis, sepsis). The clinical and sociodemographic characteristics of the patients from whom the cost information was extracted are presented in Table II.

The cost derivation follows a bottom-up approach based on the following formula: Number of " $\mathrm{A}$ " unit's $\mathrm{x}$ costs per unit " $\mathrm{A}$ " = Total cost of " $\mathrm{A}$ " units. Inpatient and outpatient costs, data of hospitalizations, and their costs were collected directly from medical invoices and health records. The direct costs considered in the analysis include: medical consultation at the emergency room, specialist referrals, chest physiotherapy, diagnosis support (laboratory, electrocardiogram, x-ray, etc.), medication (oxygen, nebulization, antibiotics, corticosteroids, bronchodilators, etc.) , medical devices, day- bed on the intensive care unit, and day-bed on the general medical ward. We used US dollars (Currency rate: US\$ $1.00=\mathrm{COP} \$$
$3,000)$ to express all costs in the study. For the evaluation of the indirect costs associated with the loss of productivity, the human capital method was used. In this, the cost-opportunity of the productivity loss at the workplace and the caregiver were assessed based on the minimum wage without including the transportation assistance for the year 2016 (U\$ 229.81 per month). Because all patients with RSV infection included were children, we assumed that at least one family member accompanied the patient permanently during hospitalization.

Table II. Sociodemographic and clinical characteristics of patients.

\begin{tabular}{|c|c|}
\hline Variable. N (\%) & $\mathrm{N}(\%)$ \\
\hline Age (months), median(ds) & $5.66(0.38)$ \\
\hline Male \%(n) & $113(58.55)$ \\
\hline Premature birth & $28(14.51)$ \\
\hline Comorbidities (CHD, neurological) & $11(5.71)$ \\
\hline Atopy & $21(10.88)$ \\
\hline SpO2.median(ds) & $88(0.93)$ \\
\hline O2 support, $\mathrm{n}(\%)$ & $178(92.33)$ \\
\hline \multicolumn{2}{|l|}{ Clinical \& laboratory parameter } \\
\hline Fever & $53(27.46)$ \\
\hline Chest indrawing & $102(52.85)$ \\
\hline Tachypnea & $30(15.54)$ \\
\hline Rhonchi & $78(40.41)$ \\
\hline Crepitation & $36(18.65)$ \\
\hline Leukocytosis (> 15.000/cm) & $31(16.76)$ \\
\hline $\begin{array}{l}\text { Increased C-reactive protein } \\
(>4 \mathrm{mg} / \mathrm{lit})\end{array}$ & $59(44.81)$ \\
\hline \multicolumn{2}{|l|}{ Chest X-ray } \\
\hline normal & $22(12.36)$ \\
\hline peribronchial thickening & $63(35.39)$ \\
\hline hyperinflation & $33(18.54)$ \\
\hline atelectasis & $5(2.81)$ \\
\hline bilateral interstitial infiltrates & $33(18.54)$ \\
\hline alveolar infiltrates & $22(12.36)$ \\
\hline Length of hospital stay, median (range) & $5.88(0.39)$ \\
\hline \multicolumn{2}{|l|}{ Complications } \\
\hline pneumonia & $23(11.92)$ \\
\hline Sepsis & $9(4.66)$ \\
\hline Atelectasis & $5(2.59)$ \\
\hline ICU & $3(1.5)$ \\
\hline
\end{tabular}

CHD: congenital heart defect, ICU: intensive care unit 
For the cost associated with transportation and food (does not include a stay), care was assumed to correspond to $50 \%$ of the day's cost for productivity loss at the workplace.

\section{Data Analysis}

Using the decision tree analysis, the cost of a patient with RSV infection was estimated. The validity of the estimates was evaluated, first by a tornado graph and the analysis of the permissible limit values to determine the variables with the greatest influence on the sensitivity analysis. In addition, a probabilistic sensitivity analysis was made using the Monte Carlo technique with a simulation of a hypothetical cohort of 10000 patients in which each parameter varied randomly according to certain distributions (beta distribution in the case of probabilities, and gamma distribution in the case of costs) according to the recommendations of Briggs; to generate $95 \%$ confidence intervals $(95 \% \mathrm{CI}) .{ }^{18}$ The Tree age 3.5 statistical package was used in all analyses.

\section{Results}

\section{Resource use and cost.}

Overall, the diagnostic tests most frequently requested by the attending physicians were hemogram in 185 (95.85\%) and chest radiography in 178 (92.22\%) patients. The medications most often prescribed were nebulized or inhaled beta 2 agonists in 108 $(55.96 \%)$, and nebulized hypertonic saline in 180 (93.26\%) patients, see Table III. Overall, the major contributors to the hospitalization costs consisted of room costs $(31.5 \%)$, drugs $(21.8 \%)$ and indirect costs (14.9\%). The medications with the highest average costs were nebulization with a hypertonic solution, systemic antibiotics, and parenteral fluids, see Table III. Diagnostic tests of both images and laboratory tests contributed to $9.32 \%$ of the costs per patient. Among these, chest radiography, blood cultures, C-reactive protein were the ones that most added to the costs per patient, see Table III.

Table III. Cost associated with RSV infection.

\begin{tabular}{lccc}
\hline & Cost/patient/day & CI 95\% \\
\hline Specialist referrals & 10.457 & 10.117 & 10.798 \\
Chest physiotherapy & 5.049 & 4.805 & 5.293 \\
Chest radiography & 2.788 & 2.643 & 2.933 \\
Others diagnostic imaging & 0.005 & 0.000 & 0.022 \\
Complete blood cell counts & 1.190 & 1.134 & 1.247 \\
RSV test & 2.892 & 2.568 & 3.215 \\
C-reactive protein and another test & 3.988 & 3.914 & 4.063 \\
Oxygen & 1.463 & 1.382 & 1.544 \\
Nebulization & 20.558 & 19.665 & 21.451 \\
Parenteral fluids & 1.367 & 1.334 & 1.399 \\
Systemic antibiotics & 1.291 & 1.193 & 1.389 \\
Systemic or inhaled corticosteroids & 0.089 & 0.000 & 0.915 \\
Bronchodilators & 0.041 & 0.036 & 0.046 \\
Medical devices & 10.664 & 10.138 & 11.190 \\
Hospital stay & 23.925 & 22.745 & 25.106 \\
The daily cost of the emergency ward & 12.833 & 12.200 & 13.467 \\
Indirect cost per day & 17.236 & 16.386 & 18.087 \\
\hline
\end{tabular}




\section{Cost of an episode of RSV infection estimated by the model.}

In the decision tree model, the mean cost per patient with RSV infection was US\$178.35 CI 95\% (30.7-541.67 US\$). The final cost estimated by the model for each outcome can be seen in Table IV. In the probabilistic sensitivity analysis, the mean cost per patient with RSV infection was stable; being only sensitive to changes in the cost of recurrent wheezing, cost of outpatient visits and cost of hospitalizations, see Figure 2. In the other variables of costs and probabilities, there were no significant variations during this analysis.

\section{Cost estimated by RSV infection in Colombia}

Taking the total of national reports of RSV infection reported to the national surveillance system; and according to the prevalence of RSV infection found in local studies ${ }^{19}$; the total cost of RSV infection in children less than 2 years in Colombia were of US \$ 64443616 per year (CI 95\% US\$11 092902 - US\$195 722 867).

\section{Discussion}

The results of this study show the high economic impact of RSV-related bronchiolitis in a tropical country. The total cost of RSV infection in children less than two years in Colombia were of US \$ 64443616 per year; this is equivalent to more than $0.7 \%$ of the total health spending in Colombia for 2018. ${ }^{20}$ Nevertheless, the proportion of cases of RSV infection in children under two years are less than $0.1 \%$ of the total of the event for any disease in Colombia. ${ }^{21}$ There is a clear imbalance between the number of patients treated and the cost that they generate, and the burden of expenses, perhaps mostly due to the use of medications or unjustified diagnostic tests. ${ }^{22,23}$

The mean cost per patient with RSV infection were US\$ 178.35 CI 95\% (30.7-541.67 US\$); this value being the most complete estimation of cost concerning this disease in any country in Latin America because we included indirect costs such as those associated with job loss

Table IV. Cost estimated by the model for each outcome.

\begin{tabular}{lccc}
\hline & Cost/patient/day & SD & Distribution \\
\cline { 1 - 2 } Not hospitalized & 171,73 & 228,92 & \\
Hospitalized without long term complications & 515,72 & 16,34 & \\
Hospitalized with long term complications & 840,52 & 189,79 & \\
\hline
\end{tabular}

\section{Tornado Diagram}

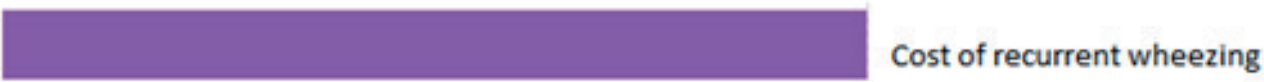

Cost of Out Patient visits

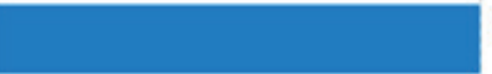

Cost of Out Patient visits

Cost of hospitalizations

Cost of emergency room visits

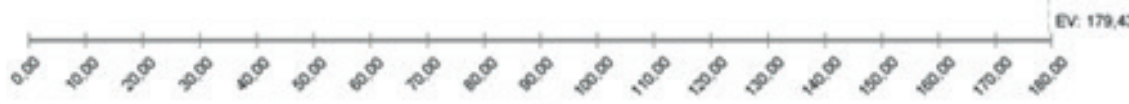

Fig. 2. Probabilistic sensitivity analysis. 
and family expenses, and the cost of long term complications of RSV infection in the first two years. These values were similar to those reported from Bangladesh (US \$ 62) 24, $^{24}$ China (US\$ 571) ${ }^{25}$, and Chile (US \$ 632). ${ }^{4}$ The study from Bangladesh was made from a social perspective, and in Chile and China using a payer perspective. As is expected, due to the greater willingness to pay, our value per patient is lower than found in developed countries (For example: United States (US \$ 2664-3799) $)^{7,26}$, United Kingdom (US \$ 3117) ${ }^{27}$ and Finland (US \$ 9551 1 $^{28}$ ). Not all previous studies were performed in the same population; most of them included the cost in children under five years and not only in the first two years. This may be difficult, in a practical sense, because the cases of RSV infection under two years are usually managed within clinical protocols of acute bronchiolitis in which the use of bronchodilators, corticosteroids, etc. are not recommended; while in older patients, especially if they have a history of wheezing episodes, are treated within asthma protocols which involve greater use of medications and diagnostic tests. ${ }^{29,30}$ In those studies that included only patients under one-year-old or preterm infants ${ }^{4}$; patients in which the initial treatment is usually more aggressive because of the risk of bacterial infection ${ }^{29}$, their cost per patient also will be higher. For this reason, the values obtained in the different studies are not comparable, and it is not appropriate to infer differences in the resources used for health care in the diverse populations studied.

In respect to the impact of RSV infections concerning morbidity, RSV is frequently linked to hospital admissions, which results in a large burden to the health care system. For example, in 2015 , around $45 \%$ of the hospitalizations and deaths occurred in RSV- in infants $<6$ months. ${ }^{2}$ Interventions to lower the prevalence and costs are deficient. No effective RSV-specific antivirals for active infection or preventive vaccines are available. RSV immune prophylaxis with targeted monoclonal antibodies (mAbs) is convenient for a limited population of high- risk infants. The number of RSV products in development highlights the need for up-to-date information to estimate the impact of vaccines, antivirals, and $\mathrm{mAb}$ on disease burden, once approved.

In our study, the mean costs per patient with RSV infection were sensitive to changes in the cost of recurrent wheezing, cost of outpatient visits, and cost of hospitalizations. It is also widely documented in other populations studied, that variables such as the cost of longterm complications and wheezing are those that have the most impact on the total cost per patient. ${ }^{30,31}$ The cost of hospitalizations may be related to the increase of inappropriate use of antibiotics for RSV infections, which directly and indirectly increases the direct costs and days of hospital stay for patients. ${ }^{32}$ This highlights the need to mitigate, through early detection in patients at risk of developing wheezing to recurrence as a strategy to be evaluated for the cost containment in this population.

Indirect costs are the costs of those resources for which no payment is made, but for which there is an opportunity cost. Guidelines for economic evaluation studies in health care recommend the inclusion of indirect costs. ${ }^{33}$ The addition of indirect costs usually had a substantial effect on the efficiency ratio, especially in pediatric diseases where hospitalization mobilizes the family, increasing the economic impact on society because of this disease. The noninclusion of such costs otherwise leads to underestimating the real cost of the disease and the effect that preventive interventions may have to reduce the frequency and duration of hospitalizations. In our study, about 15\% of the cost generated by the RSV infection is attributable to indirect costs. If we take into account that in Colombia there are about 361 332 annual cases of low acute respiratory infection due to RSV, according to the national epidemiological surveillance registries ${ }^{34}$, and in our study the indirect costs are 17 dollars per day of hospitalization; this expense they represent more than 6 million dollars per day for our society. This fact highlights the impact that 
preventive strategies may have on infections such as this one to reduce the opportunity cost and improve efficiency in the prioritization and allocation of health resources.

Our study has the following limitations: there may be differences in the costs used in this study when compared with the costs of other hospitals in Colombia. However, there is adequate adherence in our country to the use of health reference cost manuals, which means that there is low variability in the rates of each of the associated direct costs between each hospital. ${ }^{35}$ All costs were subjected to probabilistic sensitivity analysis, and none of them showed a significant change in the result of the study. Likewise, the probabilities were not obtained directly from the Colombian population. However, they were extracted from the Latin American population, and were also evaluated in the probabilistic sensitivity analysis. Likewise, the time horizon of this study is 2 years, no temporary discount value was included in the costs due to the short follow-up time of the modeled cohort. Constant discount rates devalue the long-term health benefits of prevention strongly and are usually not recommended for modeling short duration acute events.

The RSV infection in Colombia generates a high economic burden on the health system. Generating comprehensive data on healthcare resource use and costs associated with RSV will help to provide valuable information for the development of cost-effectiveness models and help guide prevention strategies against RSV.

\section{Acknowledgment}

None to declare.

\section{Ethical approval}

The study protocol and their informed consent was reviewed and approved by the Institutional Review Board of Clinica Somer (No 281015) and the University of Antioquia (No 18/2015).

\section{Author contribution}

The authors confirm contribution to the paper as follows: study conception and design: JAB, DG, DS; data collection: JAB, DG, DS ; analysis and interpretation of results: JAB, DG, DS ; draft manuscript preparation: JAB, DG, Author. All authors reviewed the results and approved the final version of the manuscript.

\section{Source of funding}

This study was supported by COLCIENCIAS (grant number 833-2015).

\section{Conflicts of interest}

The authors declare no conflict of interest.

\section{REFERENCES}

1. Shi T, McAllister DA, O’Brien KL, et al; RSV Global Epidemiology Network.Global, regional, and national disease burden estimates of acute lower respiratory infections due to respiratory syncytial virus in young children in 2015: a systematic review and modelling study. Lancet 2017; 390: 946-958.

2. Nair H, Nokes DJ, Gessner BD, et al. Global burden of acute lower respiratory infections due to respiratory syncytial virus in young children: a systematic review and meta-analysis. Lancet 2010; 375: 1545-1555.

3. Drysdale SB, Sande CJ, Green CA, Pollard AJ. RSV vaccine use--the missing data. Expert Rev Vaccines 2016; 15: 149-152.

4. Zepeda TJ, Vasquez ZJ, Delpiano ML. Direct costs of low respiratory infection due to RSV in children under one year. Rev Chil Pediatr 2018; 89: 462-470.

5. Kramer R, Duclos A, Lina B, Casalegno JS; VRS study group in Lyon. Cost and burden of RSV related hospitalisation from 2012 to 2017 in the first year of life in Lyon, France. Vaccine 2018; 36: 6591-6593.

6. Mac S, Sumner A, Duchesne-Belanger S, Stirling R, Tunis M, Sander B. Cost-effectiveness of Palivizumab for Respiratory Syncytial Virus: a systematic review. Pediatrics 2019; 143: e20184064.

7. Paramore LC, Ciuryla V, Ciesla G, Liu L. Economic impact of respiratory syncytial virus-related illness in the US: an analysis of national databases. Pharmacoeconomics 2004; 22: 275-284. 
8. Barbosa Ramirez J, Pulido Dominguez P, Rey Benito G, Mendez Rico J, Castellanos J, Páez Martinez A.Human respiratory syncytial virus and metapneumovirus in patients with acute respiratory infection in Colombia, 2000-2011. Rev Panam Salud Publica 2014; 36: 101-109.

9. Barton P, Bryan S, Robinson S. Modelling in the economic evaluation of health care: selecting the appropriate approach. J Health Serv Res Policy 2004; 9: $110-118$

10. Bereza BG, Machado M, Papadimitropoulos M, Sproule B, Ravindran AV, Einarson TR. A markov model approach assessing the cost of illness of generalized anxiety disorder in Canada. Neurol Ther 2012; 1: 1.

11. Shah NT, Wright KN, Jonsdottir GM, Jorgensen S, Einarsson JI, Muto MG. The feasibility of societal cost equivalence between robotic hysterectomy and alternate hysterectomy methods for endometrial cancer. Obstet Gynecol Int 2011; 2011: 570464.

12. Furiak N, Klein RW, Gahn JC, Camber SB, Summers $K$. Use of a decision tree model to estimate the economic benefits of reducing schizophrenia illness relapse. Value Health 2013; 16: A62-A63.

13. Barbosa J , Parra B, Alarcon L, Quinones FI, Lopez E, Franco MA. Prevalencia y periodicidad del virus sincitial respiratorio en Colombia. Rev Acad Colomb Cienc Ex Fis Nat 2017; 41: 435-446.

14. Cenco Nacional de Poblacion Y ViviendaCNPV-2018. Proyecciones de Poblacion.at: https:// www.dane.gov.co/index.php/estadisticas-por-tema/ demografia-y-poblacion/proyecciones-de-poblacion (Accessed on June 4, 2021).

15. Henderson J, Hilliard TN, Sherriff A, Stalker D, Al Shammari N, Thomas HM. Hospitalization for RSV bronchiolitis before 12 months of age and subsequent asthma, atopy and wheeze: a longitudinal birth cohort study. Pediatr Allergy Immunol 2005; 16: 386-392.

16. Ferolla FM, Hijano DR, Acosta PL, et al. Macronutrients during pregnancy and lifethreatening respiratory syncytial virus infections in children. Am J Respir Crit Care Med 2013; 187: 983990.

17. Colombia. Ministerio de Salud y Protección Social, Universidad de Antioquia. Guía de Práctica Clínica (GPC) Para la evaluación del riesgo y manejo inicial neumonía en niños y niñas menores de 5 años y bronquiolitis en niños y niñas menores de 2 años. Sistema General de Seguridad Social en Salud-Colombia. Para uso de profesionales de la salud 2014-Guía No.42 Available at: (PDF) Colombia. Ministerio de Salud y Protección Social, Universidad de Antioquia. Guía de práctica clínica para la evaluación del riesgo y manejo inicial de la neumonía en niños y niñas menores de 5 años y bronquiolitis en niños y niñas menores de 2 años. 2014 Guía No. 42. Guia completa (researchgate.net) (Accessed on June 4, 2021).

18. Briggs A, Gray A. The distribution of health care costs and their statistical analysis for economic evaluation. J Health Serv Res Policy 1998; 3: 233-245.

19. Pineros JG, Baquero H, Bastidas J, et al. Respiratory syncytial virus infection as a cause of hospitalization in population under 1 year in Colombia. J Pediatr (Rio J) 2013; 89: 544-548.

20. Ministerio de Salud y Protección Social. Gasto de Salud en Colombia 2018 Available from: https://www.minsalud.gov.co/proteccionsocial/ Financiamiento/Paginas/indicadores_generales. aspx.(Accessed on June 04, 2021).

21. Ministerio de Salud y Protección Social. Atenciones en Salud 2019.Available from: https://www.sispro. gov.co/central-gestion-del-conocimiento/Pages/ construya-su-consulta-afiliaciones-en-salud.aspx. (Accessed on May 20, 2019).

22. D Sinisterra, Buendía J. Cost illness of RSV infection in Colombia. Value Health 2017; 20: A923-A924.

23. Rodriguez-Martinez CE, Sossa-Briceno MP, CastroRodriguez JA.Direct medical costs of RSV-related bronchiolitis hospitalizations in a middle-income tropical country. Allergol Immunopathol (Madr) 2020; 48: 56-61.

24. Bhuiyan MU, Luby SP, Alamgir NI, et al. Costs of hospitalization with respiratory syncytial virus illness among children aged $<5$ years and the financial impact on households in Bangladesh, 2010. J Glob Health 2017; 7: 010412.

25. Zhang T, Zhu Q, Zhang X, et al. Clinical characteristics and direct medical cost of respiratory syncytial virus infection in children hospitalized in Suzhou, China. Pediatr Infect Dis J 2014; 33: 337-341.

26. Pelletier AJ, Mansbach JM, Camargo CA Jr. Direct medical costs of bronchiolitis hospitalizations in the United States. Pediatrics 2006; 118: 2418-2423.

27. Greenough A, Alexander J, Burgess S, et al. Health care utilisation of prematurely born, preschool children related to hospitalisation for RSV infection. Arch Dis Child 2004; 89: 673-678.

28. Heikkinen T, Ojala E, Waris M. Clinical and socioeconomic burden of respiratory syncytial virus infection in children. J Infect Dis 2017; 215: 17-23.

29. Amand C, Tong S, Kieffer A, Kyaw MH. Healthcare resource use and economic burden attributable to respiratory syncytial virus in the United States: a claims database analysis. BMC Health Serv Res 2018; 18: 294. 
30. Palmer L, Hall CB, Katkin JP, et al. Respiratory outcomes, utilization and costs 12 months following a respiratory syncytial virus diagnosis among commercially insured late-preterm infants. Curr Med Res Opin 2011; 27: 403-412.

31. Sigurs N, Bjarnason R, Sigurbergsson F, Kjellman B. Respiratory syncytial virus bronchiolitis in infancy is an important risk factor for asthma and allergy at age 7. Am J Respir Crit Care Med 2000; 161: 15011507.

32. Taylor S, Taylor RJ, Lustig RL, et al. Modelling estimates of the burden of respiratory syncytial virus infection in children in the UK. BMJ Open 2016; 6: e009337.
33. Jacobs P, Fassbender K. The measurement of indirect costs in the health economics evaluation literature. A review. Int J Technol Assess Health Care 1998; 14: 799-808.

34. Instituto, Nacional, Salud d. Infeccion respiratoria aguda en Colombia 2017 [05/07/2019]. Available at: https://www.ins.gov.co/buscador-eventos/ Informesdeevento/Informe \%20IRA \%20Final $\% 20$ 2017.pdf(Accessed on June 4, 2021).

35. DECRETO 2423 DE 1996 (diciembre 31) Diario Oficial No. 2.961, del 20 de enero de 1997. Available at: https:/www.minsalud.gov.co/sites/rid/Lists/ BibliotecaDigital/RIDE/DE/DIJ/Decreto-2423de-1996.pdf (Accessed on June 04, 2021). 